\title{
No Coil® Placement in Patients Undergoing Left Hemicolectomy and Lower Anterior Resection for Colorectal Cancer: Efficacy, Tolerability and Differences.
}

Michele Ammendola ( $\nabla$ michele.ammendola@unicz.it)

Universita degli Studi Magna Graecia di Catanzaro https://orcid.org/0000-0001-8043-6100

Michele Ruggiero

Universita degli Studi Magna Graecia di Catanzaro Scuola di Medicina e Chirurgia

Carlo Talarico

Universita degli Studi Magna Graecia di Catanzaro Scuola di Medicina e Chirurgia

Riccardo Memeo

Ospedale "F. Miulli" di Acquaviva

Giorgio Ammerata

Universita degli Studi Magna Graecia di Catanzaro Scuola di Medicina e Chirurgia

Antonella Capomolla

Universita degli Studi Magna Graecia di Catanzaro Scuola di Medicina e Chirurgia

Rosalinda Filippo

Universita degli Studi Magna Graecia di Catanzaro Scuola di Medicina e Chirurgia

\section{Roberto Romano}

Universita degli Studi Magna Graecia di Catanzaro Scuola di Medicina e Chirurgia

\section{Socrate Pallio}

Universita degli Studi di Messina

Giuseppe Navarra

Universita degli Studi di Messina

\section{Severino Montemurro}

Universita degli Studi Magna Graecia di Catanzaro Scuola di Medicina e Chirurgia

Giuseppe Currò

Universita degli Studi Magna Graecia di Catanzaro Scuola di Medicina e Chirurgia

\section{Technical innovations}

Keywords: No coil, colo-rectal cancer, anastomotic leak, left hemicolectomy, anterior resection

Posted Date: July 27th, 2020 
DOI: https://doi.org/10.21203/rs.3.rs-48501/v1

License: (c) (1) This work is licensed under a Creative Commons Attribution 4.0 International License. Read Full License 


\section{Abstract}

Background. Colorectal cancer (CRC) is the most common type of the gastrointestinal tract. Anastomotic leak (AL), is the most important complication of colorectal surgery. In this retrospective study, we evaluated efficacy and tolerability of transanal tube $\mathrm{No}^{\mathrm{Coi}}{ }^{\circledR}$ in patients with $\mathrm{CRC}$ undergoing low anterior resection (LAR) and left hemicolectomy (LC).

Methods. Thirty-eight patients operated by the same team for primary colorectal adenocarcinoma were collected, 24 LC and 14 LAR. Neither defuncioning stoma nor neoadjuvant therapy not was administered to anyone patients. No Coil ${ }^{\circledR}$ was placed immediately after the end of surgical treatment.

Results. In our experience, LC was performed in 24 individuals (63\%), LAR in 14 individuals (37\%). AL was evident in $2,63 \%$ of the sample; no statistical difference was found in AL occurrence between LC and LAR with only one patient in LAR group.

Conclusion. With our preliminary data, we suggest that No Coil ${ }^{\circledR}$ placement can be considered as a valuable procedure assisting colorectal surgery, but further studies are required to confirm and enlarge actual evidence.

\section{Introduction}

Colorectal cancer (CRC) is the most common type of cancer of the gastrointestinal tract [1] with a predicted prevalence up to 2.5 million in 2035 [2].

During 2018, 704,000 new cases of rectal cancer (RC) have been reported [3] for which low anterior resection (LAR) remains the cornerstone of curative intent treatment, providing the best results in terms of quality of life [4]. On the other hand, left colon cancer, that affects splenic flexure, descending colon and sigma, is also frequent with 138,377 of new cases in the 2014 [5] and left hemicolectomy (LC) is the surgical technique of choice required for the best oncological result.

The number of this procedures has increased thanks to advances in surgical techniques allowing an intestinal anastomosis to be feasible and safe [6]. Nevertheless, this surgical step remains a critical point in terms of tolerability and post-operative complications. Anastomotic leak (AL), defined as a defect of the intestinal wall occurring in the anastomotic site that leads to a communication between the intra- and extraluminal compartments [7] is the most important complication of colorectal surgery [8]. There is no consensus about the prevalence of $\mathrm{AL}$, since it varies according to the site of anastomosis, with colocolonic leak frequency being up to $0-9 \%$, and colorectal and coloanal leak rising to $20 \%$ [7-9].

Another important complication is prolonged post-operative ileus (PPOI), affecting up to $10 \%$ of patients undergoing colorectal surgery [10]. PPOI is defined as temporarily impaired gastrointestinal motility following surgery [11] and it is clinically evident with the absence of flatus and stools for at least five days following open abdominal surgery [12]. Both flatus and stools absence are the results of a 
combination of multiple factors, cause the increase of intraluminal rectal pressure and constitute a common final pathway to the AL [13].

To date, defunctioning stoma is the election procedure to prevent $A L$ to take place after lower anterior resection [14]. Nerveless, this procedure is burdened by several complications such as longer hospital stay, reversal procedure, greater inpatient costs, permanent stoma, stoma-related complications, and patient discomfort $[14,15]$. Given the strong evidence showing that the increased intraluminal rectal pressure is among the major contributors to $\mathrm{AL}$ [16-19] in recent years several endorectal devices (e.g. transanal tube cuff rectum, drainage tube, silicone transanal tube) $[13,20,21]$, meant to decrease endorectal pressure, have been proposed as a promising alternative to defunctioning stoma.

No Coil $\AA^{\circledR}$ is a transanal silicone stent that allows endorectal decompression and is used in low anastomosis [13].

According to recent studies, No Coil may be promising in the prevention of AL related complications, in addition to show good feasibility, cost-effectiveness and favorable patients' quality of life after treatment. However, evidence about No Coil implementation in the surgical treatment of CRC is limited to few studies, and definitive conclusions in term of efficacy and tolerability cannot be drawn. Moreover, these studies examined No Coil after LAR approach, and evidence about its efficacy after LC is still missing [13, 22].

The aim of present study was to extend actual knowledge about No Coil efficacy and tolerability in patients with CRC undergoing LAR or LC. To this extent, hospital stay, PPOI and AL events were examined and compared according to the surgical treatment. Given the exploratory nature of the study, no definite hypothesis was postulated.

\section{Materials And Methods.}

Present study took place at the Science of Health Department, Digestive Surgery Unit, University "Magna Graecia" Medical School, "Mater Domini" Hospital of Catanzaro, Italy and retrospectively examined hospital electronic medical records of patients diagnosed with CRC that underwent elective surgical intervention between January 2017 and January 2020. Diagnosis of left colon cancer (splenic flexure, descending colon and sigma) and rectum cancer, No Coil@ placement (Sapi Med Spa, Alessandria, Italy) and valid consent were considered inclusion criteria. From the initial sample, only cases operated by the same team were selected to reduce inter-operator reliability bias, resulting in thirty-eight eligible cases.

All participants were screened with Serum Carcino Embryonic Antigen (CEA) and Carbohydrate Antigen 19.9 (CA-19.9) blood level measurement, chest X-ray film, total body computed tomography (TBCT), colonoscopy and histopathological grading was performed according to American Joint of Committee on Cancer (AJCC) 8th Edition (Table 1). Surgical approach was defined according to European consensus conference [23] and The Tripartite Consensus Conference on Definitions for Anorectal Physiology and Rectal Cancer [24]. Both type of procedure (LC and LAR) were conducted in open surgery, complete 
mobilization of the splenic flexure whit end to end isoperistaltic anastomotic was performed at all participants. Neither defuncioning stoma nor neoadjuvant therapy not was administered to anyone patients.

No Coil silicone tube had the following characteristics: length of $60-80 \mathrm{~mm}$, thick $2 \mathrm{~mm}$, and diameter of $20 \mathrm{~mm}$ (Fig. 1). No Coil was placed immediately after the end of surgical treatment, inserted through the anal sphincter and stabilized 6-8 $\mathrm{cm}$ far from anus through two stitches, then removed on the seventh postoperative day if no signs of leakage occurred (Fig. 1).

Post-operative AL and PPOI events were recorded during hospital stay. AL was evaluated according to Clavien-Dindo classification [25-26].

PPOI was considered to occur when flatus and stools were absent for at least 5 days following the open abdominal surgical intervention [27]. Hospital stay was coded in days starting from the admission to the surgical department.

All procedures included in the protocol complied with the ethical standards of Helsinki Declaration and according to the Guideline for Good Clinical Practice. The Human Investigation Committee (IRB) of University "Magna Graecia” Medical School, "Mater Domini" Hospital, approved this study (Protocol N 182, 18 June 2020).

A valid, informed consent for elective surgery, as well as for the collection, managing and manipulation of medical records for scientific aims was acquired before any further step took place.

\section{Statistical analysis}

Statistical analyses were performed using the Statistical Package for the Social Sciences (SPSS) version 21.0 (SPSS 21.0; SPSS Inc., Chicago, IL, United States). Descriptive statistics included frequencies and percentages, and means and standard deviations, as appropriate. Differences between patients undergoing LC and LAR were subsequently explored through $\chi 2$ for categorical variables (AL and PPOI) and T-test for continuous variables (hospital stay). Linear and forward-stepwise logistic regression model were run to identify which variables among gender, age and type of intervention were associated with outcomes variables. Significance level was set at $p<0.05$.

\section{Results}

Twenty participants were female (52.6\%), mean age was 72,6 $\pm 6,4$. LC was performed in 24 individuals (63\%), LAR in 14 individuals (37\%). Table 2 shows demographics and comparison in outcome variables between groups. There were no differences in gender and age distribution according to the type of intervention. Mean hospital stay was $13 \pm 4.7$ days in patients undergoing LC, and $10.6 \pm 2.6$ days in patients undergoing LAR, with no significance between groups. PPOI was diagnosed in $20.8 \%$ and $28.6 \%$ of individuals undergoing LC and LAR, respectively. AL was evident in 1 patient ( $2.63 \%$, LAR group) of the 
sample; no statistical difference was found in AL occurrence between LC and LAR $(X 2=1.761 ; p=0.180)$. Results from linear regression showed that only age was significantly associated with hospital stay, explaining $8 \%$ of the variance $\left(R^{2}=0.084 ; F=4.412 ; B=.213, t=2.101 ; p=0.043 ; 95 \% \mathrm{Cl} 0.007-0.418\right)$. Age was significantly associated with PPOI ( 2 Loglikeli-hood $=33.073$; Nagelkerke $\mathrm{R}^{2}=0.302 ; \mathrm{OR}=$ $1.334 ; \mathrm{p}=0.023 ; 95 \% \mathrm{Cl} 0.040-0.537$ ) such that one-point increase in age indicates $33 \%$ increase in the odds of PPOI. Given that AL occurred in only one participant (LAR group), logistic regression was not run.

\section{Discussion}

This study sought to examine efficacy and tolerability of No Coilß in patients undergoing LAR or LC procedures for CRC.

PPOI, conceivable with gastrointestinal dysmotility occurring during post-operative time [11] was evident in $20.8 \%$ and $28.6 \%$ of individuals undergoing respectively LC and LAR. These estimates were slightly lower than the ones reported in a recent systematic review and meta-analysis, where PPOI after rectal resection is estimated to be $30.9 \%$ [30] and with respect to $27 \%$ found by Mao and colleagues after colectomy plus defunctioning stoma [29].

In present study, only one patient over 14 experienced AL complication (7.14\% in LAR group), with an overall estimate of $2.63 \%$ in the entire sample. To the best of our knowledge, only one study explored so far the efficacy of No Coil in assistance to CRC surgery. In this study, Montemurro and colleagues examined AL prevalence in a sample of 184 patients undergoing elective total or subtotal proctectomy with low-lying anastomosis and found slightly lower AL estimates 4.8\% [13]. Two randomized trial evaluated the use of transanal stent other than No Coil, that are meant to act similarly, although structurally different from it. Amin and colleagues examined the occurrence of AL after LAR plus transanal stent, compared to TAU (defunctioning stoma) and showed anastomotic leakage in three of 41 (about 7\%) [18]. Coversely, Bulow and colleagues found that transanal stent was not superior to defunctioning stoma in preventing the risk of AL after LAR (about 10.7\%) [19]. Although No Coil does not abolish the risk of AL, it may be a valuable alternative after colorectal cancer in selected patients. Accordingly, it has been demonstrated that it reduces the intraluminal rectal pressure from $13.8+$ $8.5 \mathrm{mmHg}$ to $4.8+3.7 \mathrm{mmHg}$ and protects the staple line to the fecal passage [13].

To date, no study has evaluated No Coil in assistance to LC. No anastomotic leakage occurred in patients undergoing LC surgery, in contrast with estimates of 2-7\% reported for LC and defunctioning stoma [13]. The Italian Colo-Rectal Anastomotic Leakage (iCral) study group recently reported a prevalence of AL in 4.92\% [28], but they included only symptomatic leaks, therefore, subclinical AL was not systematically diagnosed. Both PPOI and AL has an impact on hospital length of stay (LOS), and contribute to an increase in hospital costs [27]. In present study, the mean of overall hospital stay was $13 \pm 4.7$ for LC and $10.6 \pm 2.6$ for LAR, with no statistical difference between the two interventions. These results are in line with Serclova and colleagues that have found a mean stay of 10.4 (7-22) days in patients operated of 
open intestinal resections with or without stoma [31]. Similar rates were also reported in a recent multicenter trial [32].

There were no differences in all outcomes according to the type of intervention plus No Coil. The only predictor of worse outcome, as revealed for PPOI and overall hospital stay, was older age, in agreement with past evidences [33-34].

This study has some limitations. The analysis was run only on participants undergoing LC/LAR plus No Coil and compared to available data referring to TAU. The inclusion of a control group (LAR/LC plus defunctioning stoma) may have furnished a direct comparison and more reliable information about the efficacy of No Coil device. AL occurred in one patient, preventing to draw conclusions about relevant variables associated with it. Sample size was small, and may have prevented to find significance between the two interventions. However, the sample was homogeneous and only interventions of the same team were selected to avoid inter-operator reliability bias.

Notwithstanding, these results may preliminarily point out No Coil placement as a valuable procedure assisting colorectal surgery, but further studies are required to confirm and enlarge actual evidence about its efficacy in preventing short- and long-term complications.

\section{Declarations}

\section{Acknowledgement}

This work received no funding. The authors declare that they have no competing interests.

\section{Statement of Ethics}

This retrospective study involving human participants was in accordance with the ethical standards of the institutional and national research committee and with the 1964 Helsinki Declaration and its later amendments or comparable ethical standards. The Human Investigation Committee (IRB) of University "Magna Graecia" Medical School, "Mater Domini" Hospital, approved this study (Protocol № 182, 18 June 2020).

\section{Conflict of Interests Statement}

The authors declare that they have no conflict of interest.

\section{Funding Sources}

All authors have no source of funding.

\section{Consent Form}

Written informed consent for publication of clinical images was obtained from the patient 
Availability of Data and Materials

The datasets used and analysed during the current study are available from the corresponding author on reasonable request

\section{Author Contributions}

Conceptualization MA, MR, SV and GC; methodology RR and CT; validation GA and AC; formal analysis RF and SP; data curation MR and GA; writing-original draft preparation MA, GC and RR; writing-review and editing RM, SP and RR; supervision GN and SV.

These authors (MA and MR) contributed equally to this work and share the first authorship.

These authors (SM and GC) contributed equally to this work and share the senior authorship.

\section{References}

[1] Karona P, Kelaidi N, Tzouganakis A et al. (2020) Colorectal Cancer : Epidemiological Data of a Prefecture in Crete. European Journal of Surgical Oncology 46(2): e85

[2] Dekker E, Tanis PJ, Vleugels JLA et al (2019) Colorectal Cancer. Lancet 19: 1467-1480

[3] Rawla P, Sunkara T and Barsouk A (2019) Epidemiology of colorectal cancer: incidence, mortality, survival, and risk factors. Prz Gastroenterol 14(2): 89-103

[4] Robin S and McLeod MD (2001) Comparison of Quality of Life in Patients Undergoing Abdominoperineal Extirpation or Anterior Resection for Rectal Cancer Ann Surg 233(2): 157-158.

[5] Ansa BE, Coughlin SS, Alema-Mensah E, Smith SA (2018) Evaluation of Colorectal Cancer Incidence Trends in the United States (2000-2014) J Clin Med 7(2):22.

[6] Han KS, Choi GS, Park JS, Kim HJ, Park SY, Jun SH (2010) Short-term Outcomes of a Laparoscopic Left Hemicolectomy for Descending Colon Cancer: Retrospective Comparison with an Open Left Hemicolectomy. J Korean Soc Coloproctol 26(5):347-353.

[7] Bosmans JW, Jongen AC, Bouvy ND, Derikx JP (2015) Colorectal anastomotic healing: why the biological processes that lead to anastomotic leakage should be revealed prior to conducting intervention studies. BMC Gastroentero/ 15:180.

[8] James Yoo, MD (2008) Laparoscopic Colorectal Surgery. Perm J 12(1): 27-31

[9] An V, Chandra R and Lawrence M (2018) Anastomotic Failure in Colorectal Surgery: Where Are We at? Indian J Surg. 80(2): 163-170 
[10] Sciuto A, Merola G, De Palma G et al (2018) Predictive factors for anastomotic leakage after laparoscopic colorectal surgery. World J Gastroentero/ 24(21): 2247-2260

[11] Chapman SJ, Pericleous A, Downey C, Jayne DG (2018) Postoperative ileus following major colorectal surgery. Br J Surg 105(7):797-810.

[12] Wolthuis AM, Bislenghi G, Fieuws S, de Buck van Overstraeten A, Boeckxstaens G, D'Hoore A (2016) Incidence of prolonged postoperative ileus after colorectal surgery: a systematic review and metaanalysis. Colorectal Dis 18(1)

[13] Montemurro S, De Luca R, Caliandro C, et al (2012) Transanal tube NO COIL $®$ after rectal cancer proctectomy. The "G. Paolo II" Cancer Centre experience. Tumori 98(5):607-614

[14] Hanna MH, Vinci A, Pigazzi A (2015) Diverting ileostomy in colorectal surgery: when is it necessary? Langenbecks Arch Surg 400(2):145-152.

[15] Yin TC, Tsai HL, Yang PF et al (2017) Early closure of defunctioning stoma increases complications related to stoma closure after concurrent chemoradiotherapy and low anterior resection in patients with rectal cancer. World J Surg Oncol 15: 80

[16] Keigley MRB and Williams NS (1999) Surgical treatment of rectal can- cer. In: Surgery of the Anus, Rectum and Colon. Pp 1119-1244, WB Saun- ders, London

[17] Sterk P, Schubert F, Günter S, Klein P (2001) Anastomosen protektion durch transanale Drainage? Klinische Erfahrung bei 50 Patienten nach Rektumresektionen mit totaler mesorektaler Exzision und tiefen Anastomosen [Anastomotic protection with a transanal tube after rectum resection and total mesorectal excision]. Zentralb/ Chir 126(8):601-604.

[18] Amin Al, Ramalingam T, Sexton R, Heald RJ, Leppington-Clarke A, Moran BJ (2003) Comparison of transanal stent with defunctioning stoma in low anterior resection for rectal cancer. Br J Surg 90(5):581582.

[19] Bülow S, Bulut O, Christensen IJ, Harling H, Rectal Stent Study Group (2006) Transanal stent in anterior resection does not prevent anastomotic leakage. Colorectal Dis 8(6):494-496.

[20] Brandl A, Czipin S, Mittermair R, Weiss S, Pratschke J, Kafka-Ritsch R (2016) Transanal drainage tube reduces rate and severity of anastomotic leakage in patients with colorectal anastomosis: A case controlled study. Ann Med Surg (Lond) 6:12-16.

[21] Ye W, Zhu Z, Liu G, et al (2019) Application of the cuff rectum drainage tube in total mesorectal excision for low rectal cancer: A retrospective case-controlled study. Medicine (Baltimore) 98(23):e15939

[22] Montemurro S, Ammendola M, Gallo G, et al (2019) Sphincter-saving proctectomy for rectal cancer with NO COIL ${ }^{\circledR}$ transanal tube and without ostoma. Clinical outcomes, cost effectiveness and quality of 
life in the elderly. Minerva Chir 74(1):19-25.

[23] van de Velde CJ, Boelens PG, Borras JM, et al (2014) EURECCA colorectal: multidisciplinary management: European consensus conference colon \& rectum. Eur J Cancer 50(1):1.e1-1.e34.

[24] Lowry AC, Simmang CL, Boulos P, et al (2001) Consensus statement of definitions for anorectal physiology and rectal cancer. Colorectal Dis 3(4):272-275.

[25] Dindo D, Demartines N, Clavien PA (2004) Classification of surgical complications: a new proposal with evaluation in a cohort of 6336 patients and results of a survey. Ann Surg 240(2):205-213.

[26] Clavien PA, Barkun J, de Oliveira ML, et al (2009) The Clavien-Dindo classification of surgical complications: five-year experience. Ann Surg 250(2):187-196.

[27] Delaney CP, Kehlet H, Senagore A et al (2006) "Clinical Consensus Update" in General Surgery. Postoperative ileus: profiles, risk factors, and definitions - a framework for optimizing surgical outcomes in patients undergoing major abdominal and colorectal surgery. Clinical Consensus Update in General Surgery [Consensus statement].

[28] Italian ColoRectal Anastomotic Leakage (iCral) study group (2019) Colorectal surgery in Italy: a snapshot from the iCral study group. Updates Surg 71(2):339-347.

[29] Mao H, Milne TGE, O'Grady G, Vather R, Edlin R, Bissett I (2019) Prolonged Postoperative lleus Significantly Increases the Cost of Inpatient Stay for Patients Undergoing Elective Colorectal Surgery: Results of a Multivariate Analysis of Prospective Data at a Single Institution. Dis Colon Rectum 62(5):631-637.

[30] Wolthuis AM, Bislenghi G, Fieuws S, de Buck van Overstraeten A, Boeckxstaens G, D'Hoore A (2016) Incidence of prolonged postoperative ileus after colorectal surgery: a systematic review and metaanalysis. Colorectal Dis 18(1):01-09.

[31] Serclová Z, Dytrych P, Marvan J, et al (2009) Fast-track in open intestinal surgery: prospective randomized study (Clinical Trials Gov Identifier no. NCT00123456). Clin Nutr 28(6):618-624.

[32] Esteban F, Cerdan FJ, Garcia-Alonso M, et al (2014) A multicentre comparison of a fast track or conventional postoperative protocol following laparoscopic or open elective surgery for colorectal cancer surgery. Colorectal Dis 16(2):134-140.

[33] Zawadzki M, Krzystek-Korpacka M, Rząca M, et al (2019) Risk factors in reoperations in colorectal surgery. Pol Przegl Chir 91(4):13-18.

[34] Pedrazzani C, Conti C, Turri G, et al (2019) Impact of age on feasibility and short-term outcomes of ERAS after laparoscopic colorectal resection. World J Gastrointest Surg 11(10):395-406. 


\section{Tables}

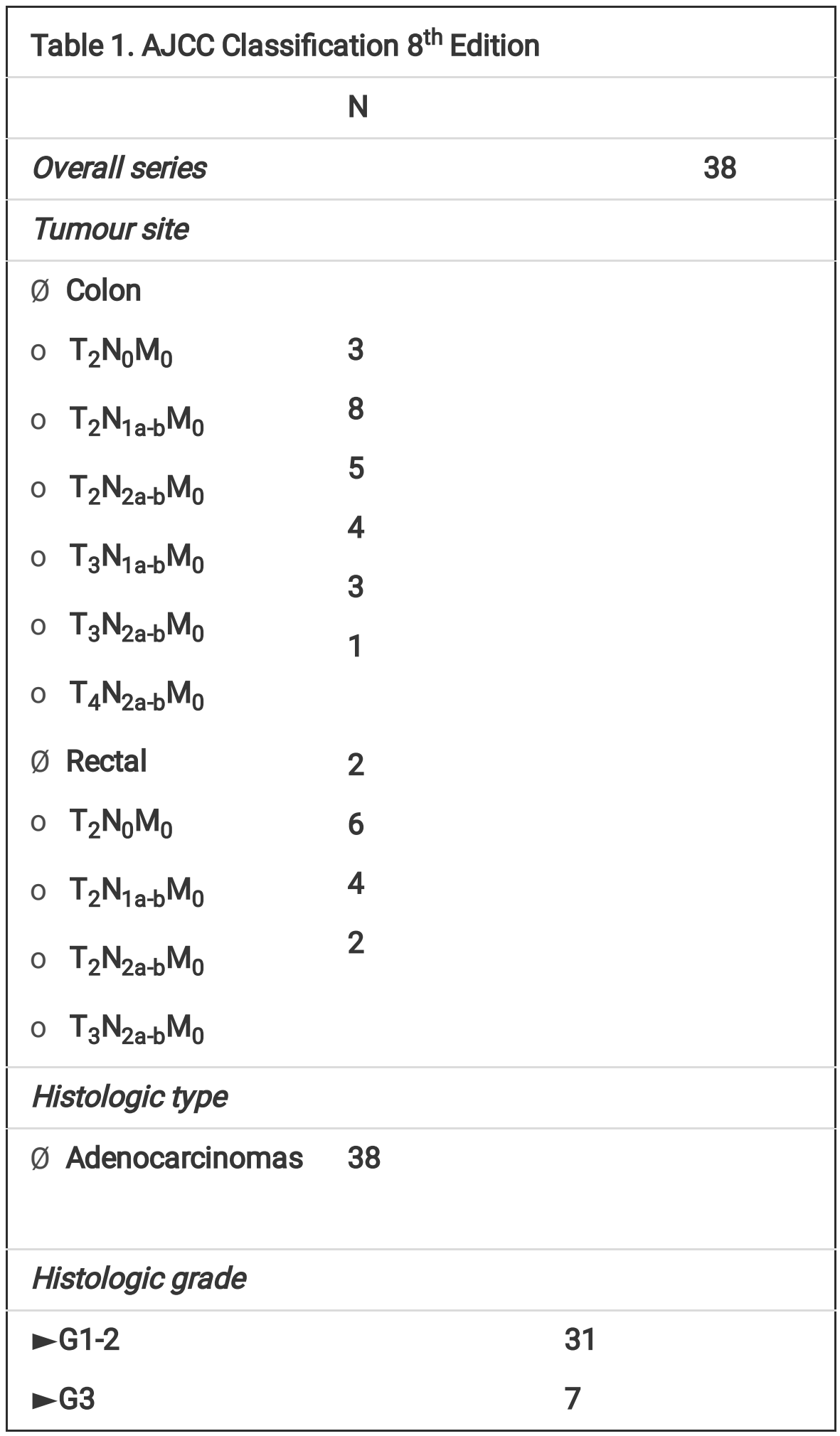


Table 2: Demographics and comparison between groups

\begin{tabular}{|c|c|c|c|c|c|c|}
\hline & \multicolumn{2}{|c|}{ LC group } & \multicolumn{4}{|c|}{ LAR group } \\
\hline & \multicolumn{2}{|c|}{$(\mathrm{N}=24)$} & \multicolumn{2}{|c|}{$(\mathrm{N}=14)$} & \multirow{2}{*}{$\begin{array}{l}\chi 2 / \mathrm{t} \\
1.280\end{array}$} & \multirow{2}{*}{$\begin{array}{l}p \\
0.209\end{array}$} \\
\hline Aget & 73.6 & 5.0 & 70.8 & 8.3 & & \\
\hline \multirow[t]{2}{*}{ genderł } & 13 & 54.2 & 5 & 35.7 & 1.208 & 0.272 \\
\hline & 11 & 45.8 & 9 & 64.3 & & \\
\hline PPOI¥ & 5 & 20.8 & 4 & 28.6 & 0.293 & 0.588 \\
\hline Hospital stay† & 13 & 4.7 & 10.6 & 2.6 & 1.724 & 0.093 \\
\hline $\mathrm{AL} \neq$ & 0 & 0 & 1 & 7.14 & 1.761 & 0.185 \\
\hline \multicolumn{7}{|l|}{ †Mean and SD } \\
\hline \multicolumn{7}{|l|}{$\ddagger \mathrm{fr}, \%$} \\
\hline \multicolumn{7}{|c|}{$\begin{array}{l}\text { LC: left hemicolectomy; LAR: low anterior resection; PPOI: prolonged post-operative ileus; AL: } \\
\text { anastomotic leak }\end{array}$} \\
\hline
\end{tabular}

\section{Figures}




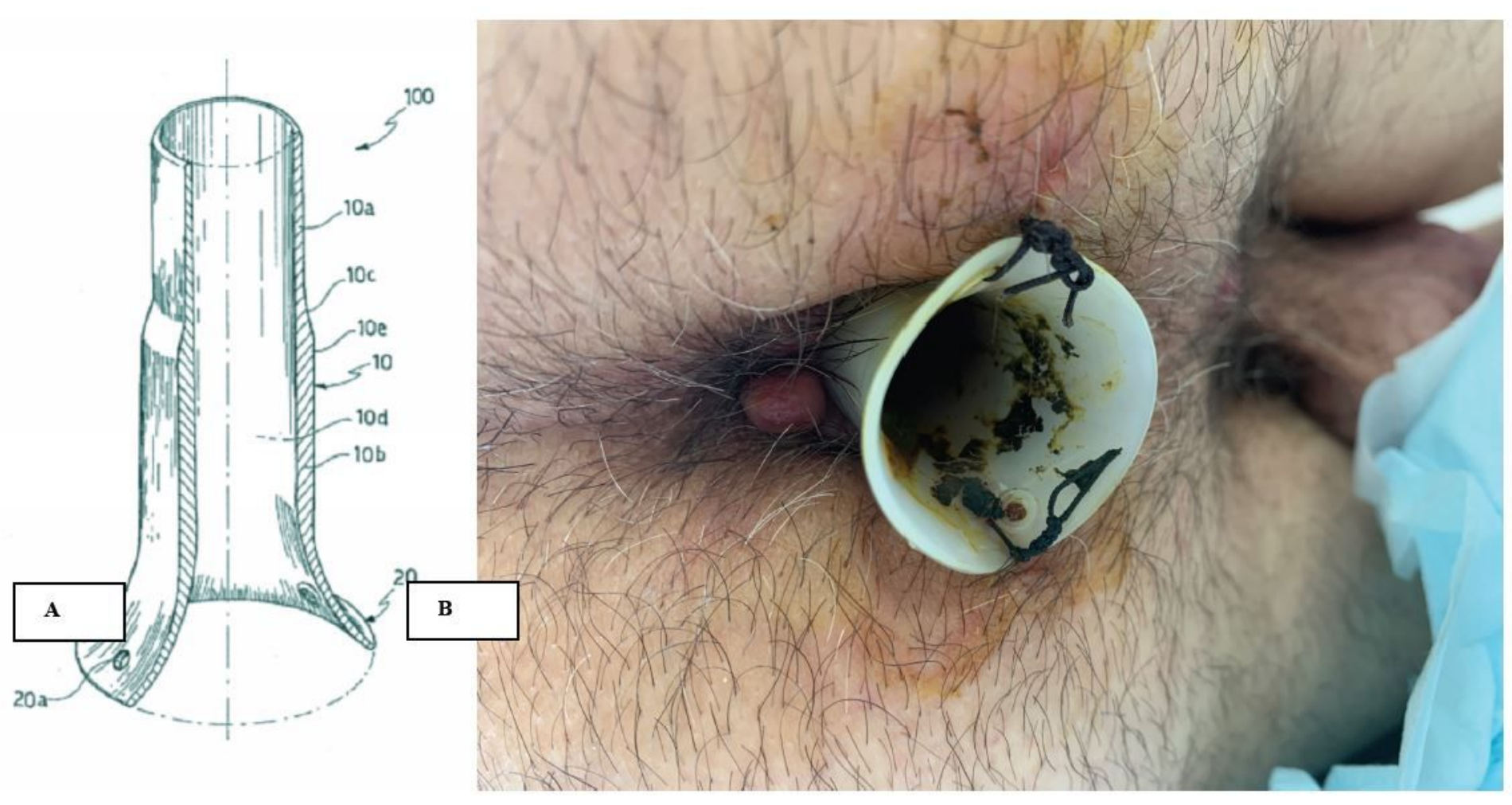

\section{Figure 1}

No Coil structure and the postoperative placement. A. length of $60-80 \mathrm{~mm}$, thick $2 \mathrm{~mm}$, and diameter of $20 \mathrm{~mm}$. B. stabilized 6-8 $\mathrm{cm}$ far from anus through two stitches. 